\title{
ESTUDIO PRELIMINAR DE LAS ALGAS DE LA ZONA DE PUCALLPA
} (DEPARTAMENTO DE LORETO)

\author{
Haydée Montoya T. \\ Departamento de Botánica, Museo de Historia Natural "Javier Prado" \\ Universidad Nacional Mayqor de San Marcos, Lima, Perú.
}

\section{SU MAR IO}

Este trabajo reporta 17 géneros y 27 especies de algos de agua dulce colectados en Pucallpa, Departamento de Loreto.

Las siguientes especies de CYANOPHYTA y CHLOROPHYTA son nuevos reportes pora la flora peruana: Chroococcus minor, Microspora tumidula, Cylindrocystis brebissonit, Closterium baillyanum, Closterium johnnsonii, Euastrum luetkemuellerii, Euastrum paulense, Cosmarium contractum, Cosmarium punctulatum, Desmidium aptogonum y Phymatodocis nordstedtiana.

\section{S U M M A R Y}

This paper reports about 17 genera with 27 species of fresh-water algoe collected at Pucallpa, Department of Loreto.

The following species of CIANOPHYTA and CHLOROPRYTA ore new records to the Peruvian flora: Chroococcus minor, Microspora tumidula, Cylindrocystis brebissonii, Closterium baillyanum, Closterium johnnsonii, Euastrum luetkemuellerii, Euastrum paulense, Cosmarium contractum, Cosmarium punctulatum, Desmidium aptogonum and Phymarodocis nordstedtiana.

\section{INTRODUCCION}

En relación al estudio de las algas colectadas en zonas próximas a la de Pucallpa, sólo encontramos referencias en los trabajos de Sánchez y Ancieta (1955), Krieger y Scott (1957), Scott, Gronblad y Croasdale (1965), Acleto (1969) y Forster (1969). Considerando esta reducida cantidad de investigaciones ficológicas de nuestra Amazonía, se realizó el presente trabajo como una contribución preliminar para estudios futuros en el campo de la ficología dulce-acuicola de nuestro país.

Este estudio se ha efectuado sobre 6 muestras colectadas en 1972, en los alrededores de la Estación Principal del Trópico del I.V.I.T.A. (Pucallpa). Incluye a 17 géneros pertenecientes a las Cianophyta y Chlorophyta, predominando entre éstas los integrantes de las algas verdes. Sobresalen en número las integrantes de la familia Desmidiaceae, los cuales, por sus exigencias biológicas, caracterizan muchos ambientes acuáticos.

Las algas identificadas suman 27 especies, de las cuales 11 son registradas por primera vez para nuestro país.

\section{MATERIAL Y METODOS}

Las muestras estudiadas fueron colectadas por los Biólogos W. Gutiérrez y H. Ortega, en unos pequeños embalses naturales presentes en la Estación Principal del Trópico del I.V.I.T.A. (Pucallpa) en 1972.

Todas las muestras fueron examinadas bajo el microscopio compuesto en preparados temporales simples, utilizándose en algunos casos una solución de anilina azul al $1 \%$ para poner de manifiesto estructuras poco notables.

Este material, catalogado y preservado en una solución de formalina al $5 \%$, forma parte de la colección ficológica del Herbario San Marcos (USM) del Museo de Historia Natural "Javier Prado" de la Universidad Nacional Mayor de San Marcos, Lima.

Las ilustraciones fueron realizadas por el autor, utilizando una cómara clara Leitz. 
LISTA SISTEMATICA DE LOS

GENEROS $Y$ ESPECIES

CYANOPHYTA

Familia Chroococcaceae

CHROOCOCCUS Naegeli

Chroococcus minor (Kuetz.) Naegeli

Familia Oscillatoriaceae

OSCILLATORIA Vaucher

Oscillatoria tenuis Agardh

Oscillatoria sp.

CHLOROPHYTA

Familia Volvocaceae

PANDORINA Bory

Pandorina morum (Muell.) Bory

Familia Microsporaceae

MICROSPORA Thuret

Microspora fumidula Hazen

Familia Cladophoraceae

PITHOPHORA Witthrock

Pithophora sp.

Familia Zygnemataceae

SPIROGYRA Link

Spirogyra sp.

Familia Mougeotiaceae

Mougeotia sp.

MOUGEOTIA Agardh

Familia Mesotaeniaceae

CYLINDROCYSTIS Meneghini

Cylindrocystis brebissonii Meneghini

Familia Gonatozygaceae

GONATOZYGON De Bary

Gonatozygon monotaenium De Bary

Familia Desmidiaceae

CLOSTERIUM Nitzch

Closterium baillyanum Bréb.

Closterium johnsonii. .W. et G. S. West

Closterium dianae Ehrenberg

Closterium kuetzingii Brebisson

Closterium libellula Focke

Closterium malmei Borge

PLEUROTAENIUM Naegeli

Pleurotaenium trabecula (Ehr.) Naegeli

Pleurotaenium nodosum (Bail). Lund

EUASTRUM Ehrenberg

Euastrum ansatum Raifs

Euastrum incavatum Josh. et Nordst.

Euastrum luetkemuellerii Ducell

Euastrum paulense Borgesen
MICRASTERIAS Agardh

Micrasterias laticeps Nordstedt

COSMARIUM Corda

Cosmarium contractum Kirchner

Cosmarium pseudoconnatum Nordstedt

Cosmarium pyramidatum Bréb.

Cosmarium punctulatum Bréb.

STAURASTRUM Meyen

Staurastrum setigerum Cleve

DESMIDIUM Agardh

Desmidium aptogonum Brébisson

Desmidium cylindricum Grev.

PHYMATODOCIS Nordstedt

Phymatodocis nordstedtiana Wolle

\section{DESCRIPCION DE LAS ESPECIES}

CYANOPHYTA
CHROOCOCCUS Naegeli
Chroococeus minor (Kuetz.) Naegeli

(Fig. 1)

Esta especie se presenta formando masas amorfas que incluyen a células esféricas o angulares por compresión, las cuales se distribuyen en forma irregular, generalmente solitarias o en pares, rara vez en grupo de 4 a 8 células como resultado de repetidas divisiones; protoplasto finamente granulado, rodeado de un mucilago hialino, los especímenes individuales miden de 5.4 a 6 micras de diámetro total.

\section{OSCILLATORIA Vaucher \\ Oscillatoria tenuis Agardh \\ (Fig. 3)}

Tricomas casi rectos en toda su extensión o ligeramente sinuosos en los extremos, solitarios o agrupados, no atenuados hacia los ápices; células cilindrico-discoidales, constrictas al nivel de las paredes transversales, de 4.5-6 micras de longitud por 6-6.9 micras de diámetro; protoplasto homogéneo, lleva una hilera de grónulos a ambos lados de las paredes transversales; célula apical hemiesférica, no capitada, de 5.4 micras de longitud.

Oscillatoria sp.

(Fig, 2)

Tricomas en su mayoría rectos, solitarios o agrupados, ligeramente atenuados 
hacia los extremos; células discoidales, de 3-3.9 micras de longitud, por 6-6.6 micras de diámetro, levemente constrictas al nivel de las paredes transversales; protoplasto con granulaciones notorias distribuídas en forma uniforme; célula apical levemente capitada.

\section{CHLOROPHYTA \\ PANDORINA Bory \\ Pandorina morum (Muell.) Bory}

(Fig. 4)

Especie colonial esférica o elíptica, de vida libre, de 34.5-40.5 micras de diámetro; generalmente constituida por 16 células piriformes o poligonales por compresión, dispuestas más o menos radialmente, de 13.5-16.5 micras de diámetro.

\section{MICROSPORA Thuret \\ Microspora tumidula Hazen \\ (Fig. 5)}

Filamentos formados por células cilindricas, de 9-15 micras de longitud por 12 micras de diámetro; pared celular notoria, pero las secciones en forma de $H$ son dificilmente distinguibles; cloroplasto laminar perforado, parietal, algunas veces ocupan toda la célula.

Se le ha clasificado como tal a pesar de que es muy difícil distinguirla de las especies: $M$. floccosa, $M$. stagnorum y $M$. willeana en condición vegetativa, sólo pueden ser diferenciados por el tamaño de los acinetos, forma de reproducción vegetativa no observada.

\section{PITHOPHORA Wittrock} Pithophora sp.

(Figs. 6 y 7 )

Filamentos ramificados, de 13.5-16 micras de diámetro; células largas, cilíndricas, de 78-240 micras de longitud; acinetos en forma de barril con el extremo libre acuminado, de 31.5-39 micras de diámetro y 57-58.5 micras de longitud.

\section{SPIROGYRA Link Spirogyra sp.}

(Fig. 8)

Filamentos de células largas, cilindricas, de 61-72 micras de diámetro por 250-405micras de longitud; pared transversal plana; 3 cromatóforos que dan 2-4 vueltas espiraladas; conjugación escaleri- forme, tubos formados por ambos gametangios; zigospora elipsoidal, con pared externa lisa y hialina, de 96-126 micras de longitud y 56-60 micras de diámetro.

\section{MOUGEOTIA Agardh Mougeotia sp. \\ (Fig. 9)}

Células vegetativas largas, cilindricas, de 9 micras de diámetro por 33 micras de longitud; cloroplasto laminar, ocupa casi toda la longitud de la célula, con numerosos pirenoides.

\section{CYLINDROCYSTIS Meneghini} Cylindrocystis brebissonii Meneghini (Fig. 10)

Células pequeñas, cilíndricas, en general 2 a 4 veces más largas que anchas, con los polos ampliamente redondeados, sin constricción media; pared celular lisa e incolora; con dos cloroplastos axiales, cada uno con un pirenoide grande.

Longitud celular: 37.5 micras; ancho: 13.5 micras; ápice: 7.5 micras.

GONATOZYGON De Bary Gonatozygon monotaeniun De Bary (Fig. 11)

Células alargadas, cilindricas, con los ápices ligeramente dilatados; pared celular menuda y densamente granulada; dos cromatóforos laminar-axiales conteniendo numerosos pirenoides.

Longitud celular: 186 micras; ancho: 12 micras; ápice: 12.6 micras.

\section{CLOSTERIUM Nitzsch Closterium baillyanum Brébisson \\ (Fig. 12)}

Células de tamaño mediano, atenuados gradual o escasamente hacia los ápices, los cuales son truncados; superficie ventral casi recta, algunas veces con una ligera concavidad; pared celular amarillenta, fina y densamente punteada, con los extremos más oscuros y donde la ornamentación es más notoria; con dos cloroplastos axiales, con numerosos pirenoides cada uno.

Longitud celular: 258 micras; ancho 33 micras; ápice: 14.2 micras. 
Closterium johnsonii W. et G. S. West

(Figs. 13 y 14)

Células grandes, con los lados de los ápices casi rectos, superficie del vértice aplanada o algo convexa; pared celular lisa, de color amarillo pálido; con dos cromatóforos axiales y numerosos pirenoides.

Longitud celular: 546 micras; ancho: 36 micras; ápice: 9 micras.

\section{Closterium dianae Ehrenberg}

(Fig. 15)

Células fuertemente curvadas, cuyo margen interno cóncavo puede ser ligeramente hinchado o no en su parte media, gradualmente atenuado hacia los ápices, los cuales son obtuso-redondeados y donde es notorio la presencia de nódulos apicales; pared celular lisa, a veces de un color amarillo pálido; dos cromatóforos axiales con varios pirenoides.

Longitud celular: 139.5 micras; ancho: 9 micras; ápice: 3 micras.

\section{Closterium kuetzingii Brébisson}

(Fig. 16)

Células relativamente grandes, casi rectas en toda su longitud, con la porción media fusiforme-lanceolada, siendo ambos márgenes igualmente convexos, atenuándose hacia los extremos, semejando a procesos setáceos; ápice levemente curvado, redondeado y a menudo hinchado; pared celular finamente estriada, de color amarillento; con dos cloroplastos axiales y varios pirenoides.

Longitud celular: 310 micras; ancho: 16.5 micras; ápice: 3 micras.

\section{Closterium libellula Focke}

(Fig. 17)

Células medianas, fusiformes, cuya longitud contiene varias veces el ancho, gradualmente atenuados del medio a los ápices, éstos con bordes truncado-redondeados; pared celular lisa e incolora; con dos cromatóforos esteloides, cada uno con una interrupción mediana.

Longitud celular: 111 micras; ancho: 25.5 micras; ápice: 10.5 micras.

\section{Closterium malmei Borge}

(Fig. 18)

Células medianas, fuertemente curvadas; pared celular pardo amarillenta, con listones bien marcados, los que terminan en hileras de puntos en los ápices de las semicélulas; dos cromatóforos axiales con numerosos pirenoides.

Longitud celular: 238 micras; ancho: 39.7 micras; ápice: 12 micras.

PLEUROTAENIUM Naegeli Pleurotaenium trabecula (Ehr.) Naegeli

(Fig. 19)

Células largas, cilíndricas; semicélulas con una inflación basal pronunciada, márgenes laterales casi rectos, con una ligera atenuación en los ápices que son redondeado-truncados, y carecen de tubérculos o dientes; pared celular densamente punteada; con dos cloroplastos con bandas irregulares y numerosos pirenoides.

Longitud celular: 763 micras; ancho basal. 34.5 micras; ápice: 27 micras; istmo: 27 micras.

\section{Pleurotaenium nodosum (Bail.) Lund (Fig. 20)}

Células solitarias, majestuosas, con una profunda constricción que divide la célula en dos semicélulas, cada una con 4 inflaciones o protuberancias gradualmente atenuadas de la base a los extremos; ápices dilatados, convexo-truncados, con una corona de 8 dientes cónicos; pared celular marrón oscuro; dos cloroplastos axiales con varios pirenoides.

Longitud celular: 417 micras; ancho basal: 72 micras; ápice: 33 micras; istmo: 36 micras.

\section{EUASTRUM Ehrenberg Euastrum ansatum Ehrenberg} (Fig. 21)

Células de aspecto más bien elongado, con marcada constricción; semicélulas piramidales, de ápices convexo-truncados, ángulos basales redondeados y los márgenes laterales no ondulados; pared celular incolora; con dos cloroplastos axiales y densos. 
Longitud celular: 61.5 micras; ancho: 33.7 micras; ápice: 15.7 micras; istmo: 9 micras.

Euastrum incavatum Josh. et Nordst. (Fig. 22)

Células pequeñas, profundamente constrictas, sinus linear, ápices convexos, con dos denticulaciones y otras dos en los márgenes laterales basales de las semicélulas; pared celular lisa e incolora; dos cromatóforos laminar axiales, con un pirenoide cada uno.

Este especimen se aproxima al ilustrado por Krieger y Scott (1957), siendo esta determinación tentativa.

Longitud celular: 36 micras; ancho: 21 micras; ápice: 15 micras; istmo: 4.5 micras.

\section{Euastrum luetkemuellerii Ducell (Fig. 23)}

Células pequeñas, anchas, con marcada constricción en su parte media, sinus linear con el extremo dilatado; semicélulas subcuadradas, ángulos basales redondeados, ápices convexo-truncados; pared celular lisa e incolora; dos cloroplastos laminar axiales con un pirenoide central cada uno.

Longitud celular: 22.5 micras; ancho: 18 micras; ápice: 14.4 micras; istmo: 8 micras.

\section{Euastrum paulense Boergesen}

(Fig. 24)

Células pequeñas con marcada constricción en su parte media, sinus linear; semicélulas con tres lóbulos basales, divididos por una incisión superficial en dos lóbulos, cada uno con sus ángulos terminados en cortas proyecciones denticulares, lóbulo polar subcuneiforme, con una incisión media profunda; pared celular lisa e incolora; dos cromatóforos laminar-axiales con un pirenoide cada uno.

Longitud celular: 32.5 micras; ancho: 24 micras; ápice: 15 micras; istmo: 7.6 micras.
MICRASTERIAS Agardh

Micrasterias laticeps Nordstedt

(Fig. 25)

Células casi tan largas como anchas, con profunda constricción media; semicélulas con tres lóbulos basales dispuestos horizontalmente, subfusiformes, con extremos bífidos, lóbulo polar ancho, fusiforme, con polos agudos; pared celular incolora y densamente porosa; un cloroplasto laminar-axial con varios pirenoides en cada semicélula.

Longitud celular: 111 micras; ancho del lóbulo basal: 141 micras; ancho del lóbulo polar: 121 micras; istmo: 21 micras.

\section{COSMARIUM Corda \\ Cosmarium contractum Kirchner}

(Fig. 26)

Células pequeñas, con constricción no muy profunda, sinus abierto, semicélula subelíptica, con el margen ventral más convexo que el dorsal; pared celular densamente punteada; dos cromatóforos laminar-axiales con un pirenoide cada uno.

Longitud celular: 23 micras; ancho: 16.5 micras; ápice: 9 micras; istmo: 10 micras.

\section{Cosmarium pseudoconnatum Nordstedt (Fig. 27)}

Células con constricción media superficial, semicélulas semejantes a hemiesferas; pared celular incolora y densamente punteada; 4 cromatóforos por semicélula, cada uno con un pirenoide.

Longitud celular: 43 micras; ancho: 34 micras; ápice: 15 micras; istmo: 28.5 micras.

\section{Cosmarium pyramidatum Brébisson (Fig. 28)}

Células medianas, profundamente constrictas en su parte media, sinus lineal, dilatado en sus extremos; semicélulas truncado-piramidales, ángulos basales redondeados; pared celular incolora $y$ densamente punteada; dos cloroplastos laminar-axiales.

Longitud celular: 66 micras; ancho: 45 micras; ápice: 20 micras; istmo: 13 micras. 
Cosmarium punctulatum Brébisson

(Figs. 29, 30 y 31)

Células pequeñas, con profunda constricción en su parte media, sinus linear, dilatado en sus extremos; semicélulas oblongo-trapezoidales, con ángulos basales redondeados y lados convexos; vista lateral de la semicélula circular con entumecimientos en la parte media de cada lado; vista apical elíptica; pared celular uniformemente granulada; dos cloroplastos axiales con dos pirenoides cada uno.

Esta especie se clasifica como tal a pesar de que los especimenes estudiados presenta invariablemente dos pirenoides por cloroplasto, contradiciendo así la literatura consultada.

Longitud celular: 29 micras; ancho: 26 micras; ápice: 12 micras; istmo: 9 micras.

\section{STAURASTRUM Meyen \\ Staurastrum setigerum Cleve \\ (Figs. 32 y 33)}

Células de tamaño pequeño, con profunda constricción, sinus abierto; semicélulas aovado-elípticas, vista por el ćpice triangular, cuyos lados presentan una ligera concavidad y los ángulos agudamente redondeados; pared celular con largas espinas punteadas, distribuidas en forma irregular alrededor de los ángulos.

Longitud celular: 37 micras; ancho: 31 micras; ápice: 12 micras; istmo: 9 micras.

DESMIDIUM Agardh

Desmidium aptogonum Brébisson

(figs. 34 y 35)

Alga filamentosa, de células medianas, con constricción moderada, sinus abierto y agudo; semicélulas con ángulos laterales redondeados, ápices cóncavos en su parte media, de modo que existe una considerable cavidad entre las células adyacentes; vista apical trirradiada, lados cóncavos y ángulos redondeados; pared celular incolora; dos cloroplastos laminar axiales, cada uno con dos pirenoides.

Longitud celular: 15.5 micras; ancho: 32 micras; istmo: 21 micras.

\section{Desmidium cylindricum Grev. \\ (Fig. 36)}

Filamentos espiralados, células con ligera constricción, ápices planos, por lo que no existe cavidad entre las células adyacentes; pared celular incolora, con 3-4 hileras horizontales de poros claramente visibles en las bases de las semicélulas; dos cromatóforos axiales, cada uno con 4 proyecciones lobuladas.

Longitud celular: 26 micras; ancho: 45-85.5 micras; ápice: $21-30$ micras; istmo: $34.5-48$ micras.

\section{PHYMATODOCIS Nordstedt Phymatodocis nordstedtiana Wolle (Figs. 37,38 y 39)}

Alga filamentosa de células rectangulares, casi tan largas como anchas, con profunda constricción, vista apical irregularmente cuadrangular; pared celular de color amarillo pálido o castaño.

El género Phymatodocis con la especie $\mathbf{P}$. nordstedtiana es registrado por primera vez para nuestro país.

\section{AGRADECIMIENTOS}

Mi sincero reconocimiento al Dr. César Acleto 0 . por sus invalorables consejos y la orientación recibida en la realización del presente estudio.

Hago extensivo mi agradecimiento al Dr. R. Ferreyra, Director del Museo de Historia Natural "Javier Prado" de la Universidad Nacional Mayor de San Marcos, por las facilidades brindadas en los laboratorios del Departamento de Botánica de dicho Museo. 


\section{REFERENCIAS BIBLIOGRAFICAS}

ACLeTO, O. C. - 1966. Algas de Agua Dulce de las Cascadas de Barranco. Publ. Mus. Hist. Nat. "Javier Prado", Serie B. 21:1-65. Lima.

ACLETO, O. C. - 1969. Dos especies de Cyanophyta nuevas que se registran para el Perú. Pub. Mus. Hist. Nat. "Javier Prado", Serie B. 23:1-8. Lima.

ACLETO, O. C. - 1971. Algas del Valle de Camaná. Est. Exp. del Camarón. Camaná. Dpto. Arequipa. Raymondiona 4:79-98. Lima.

ALDAVE, P. A. - 1970. Nuevos Géneros de Desmidias para el Perú. Bol. Soc. Bot. La Libertod:2(1-2): 1-26, 3 lóms., Trujillo. Perú.

BICUDO, C. E. M. y ROSA M. BICUDO - 1970. Algas de Aguas Continentais Brasileiras. Chove ilustrada para identificacao de Generos. Funbec. Soo Paulo. Brosil.

DESIKACHARY, T. V. - 1959. Cyanophyta. Indian Council of Agri. Res., 638 p., 139 pls., New Delhi.

FORSTER, K. - 1969. Amazonische Desmidieen I. Teil: Area de Santarem. Amazoniona 2(2): 1-116, Taf:56. Kiel.

GRONBLAD, R., G. A. PROWSE and A. M. SCOTT - 1958. Sudanese Desmids. Act. Bot. Fenn. 58. Helsinki.

GRONBLAD, R., A. M. SCOTT and H. CROASDALE - 1964. Desmids from Uganda and Lake Victorio. Act. Bot. Fenn. 66. Helsinki.

GRONBLAD, R. and H. CROASDALE - 1971 Desmids from Namibia (S W Africa). Act. Bot. Fenn. 93. Helsinki.
GUARRERA, S. A. et al. - 1972. Fitoplaneton de las aguas superficiales de la Provincia de Buenos Aires. II complejo Lagunar Salado Grande. Encodenadas del oeste y Encadenadas del Sur. Rev. Mus. La Plata. N. Sr. Bot. t. 12, págs. 161-219. Argentina.

HALPERIN, DELIA R. de - 1967. Cionoficeos Marinas de Puerto Deseado (Prov. Sto. Cruz, Argentina) II, Darwiniana 14(2-3):273-338, 14 lóms. Bs. As. Argentina.

KRIEGER, W. \& A. M. SCOTT - 1957. Einege Desmidioceen aus Perú. Hidrobiologia. $9(2-$ 3): 126-144. Den Haag.

LACOSTE, ELSA N. de - 1963. II Desmidiaceos de Sierra de La Ventana (Prov. de Buenos Aires). Darwiniana 12(4), págs. 575-597.

PRESCOTT, G. M. - 1951. Algoe of the Westem Great Lakes Area. Cronbrook Inst. Sc., 946 págs., 136 pls. EE.UU.

SANCHEZ, J. y F. ANCIETA - 1946. Limnologío y Piscicultura en la Selva Peruana: Plancton de la Cocha Zapote., Pub. Serv. Piscicultura del Oriente. Iquitos. Minst. Agri.

SCOTT, A. M., R. GRONBLAD and H. CROASDALE - 1965. Desmids from Amazon Basin, Brasil. Act. Bot. Fenn. 69. Helsinki.

TAFT, C. E. and C. W. TAFT - 1971. The Algoe of Western Lake Erie. Bull. Ohio Biological Survey, N. Srs. 4(1). Publ. Ohio State Univ. Columbus. Ohio.

YACUBSON, SARA - 1956. Géneros de Desmidiaceae de la República Argentina. Univ. Bs. As. Sr. Bot. 1(1):1-68. Argentino. 
Fig. I Chroococeus minor (Kuetz.) Noegeli, varias colonias; Fig. 2 Oscillatoria sp., porción terminal del tricoma; Fig. 3 Oscillatoria tenuis Agardh, porción terminal del tricomo; Fig. 4 Pandorina morum (Muell.) Bony, colonia; Fig. 5 Microspora tumidula Hozen; Fig. 6 Pithophora sp., rama con acinetos terminales; Fig. 7 Pithophora sp. ramificación y acinetos; Fig. 8 Spyrogyra sp., filomentos en conjugación escaleriforme.

Fig. 9 Mougeotia Sp.; Fig. 10 Cylindrocystis brebissonii Meneghini; Fig. 11 Gonatozygon monotaenium De Bary; Fig. 12 Closterium baillyanum Bréb.; Fig. 13 Closterium johnsonii W. et G. S. West; Fig. 14 Closterium johnsonii W. et G. S. West, porción terminal de la célula; Fig. 15 Closterium dianae Ehrenber; Fig. 16 Closterium kuetzingii Brébisson; Fig. 17 Closterium libellula Focke; Fig: 18 Closterium malmei Borge.

Fig. 19 Pleurotaenium trabecula (Ehr.) Naegeli, hemicélula; Fig. 20 Pleurotaenium nodosum (Bail.) Lund, hemicélulai Fig. 21 Euastrum ansatum Ehrenberg; Fig. 22 Euastrum incavatum Josh. \& Nordst.; Fig. 23 Euastrum luetkemuellerii Ducell; Fig 24 Euostrum paulense Boergesen; Fig. 25 Microsterias laticeps Nordstdt; Fig. 26 Cosmarium contractum Kirchner; Fig. 27 Cosmarium pseudoconnatum Nordstedt; Fig. 28 Cosmarium pyramidatum Bréb.; Fig. 29 Cosmarium punctulatum Bréb.; Fig. 30 Cosmarium punctulatum Bréb., vista apical; Fig. 31 Cosmarium punctulatum Bréb., Visto loteral.

Fig. 32 Staurastrum setigerum Cleve, visto apical; Fig. 33 Staurastrum setigerum Cleve, visto frontal; Fig. 34 Desmidium aptogonum Brébisson, vista apical; Fig. 35 Desmidium aptogonum Brébisson, colonia filamentoso; 'Fig. 36 Desmidium cylindricum Grev., colonia filomentosa; Fig. 37 Phymatodacis nordstedtiona Wolle, células en reproducción; Fig. 38 Phymatodocis nordstedtiana Wolle, vista opical; Fig. 39 Phymatodocis nordstedtiana Wolle, colonia filomentoso.

Fig. 40 Oscillatoria tenuis Agardh; Fig. 41 Oscillatoria sp.; Fig. 42 Pandorina morum (Muell.) Bory; Fig. 43 Cylindrocystis brebissonii Meneghini; Fig. 44 Closterium diance Ehrenberg; Fig. 45 Pleurotaenium nodosum (Bail.) Lund; Fig. 46 Cosmarium pseudoconnatum Nordstedt; Fig. 47 Cosmarium pyramidatum Bréb.; Fig. 48 Cosmarium punctulatum Bréb.

Fig. 49 Phymatodocis nordstedtiane Wolle; Fig. 50 Desmidium cylindricum Grev; Fig. 51 Desmidium aptogonum Brébisson. 


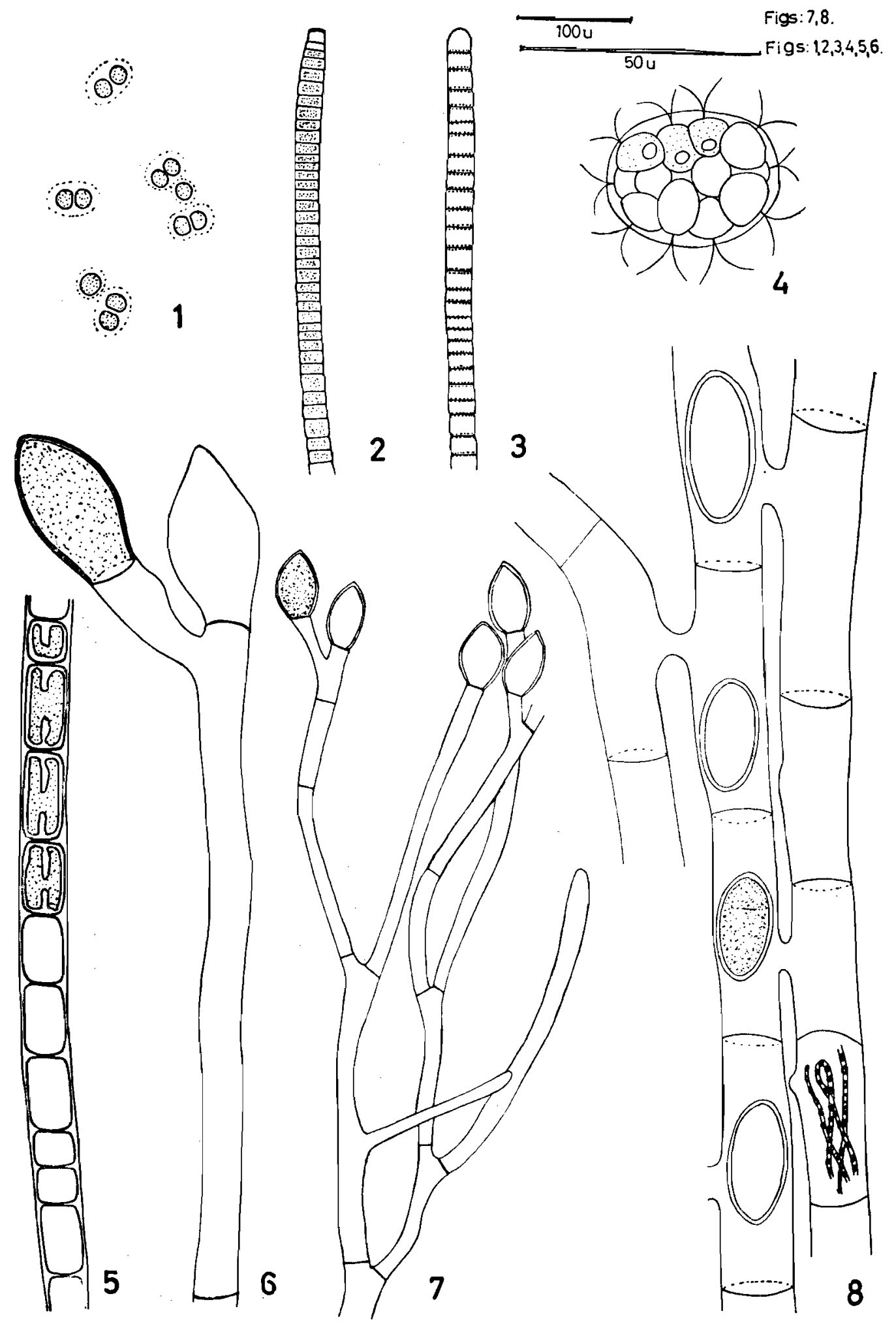




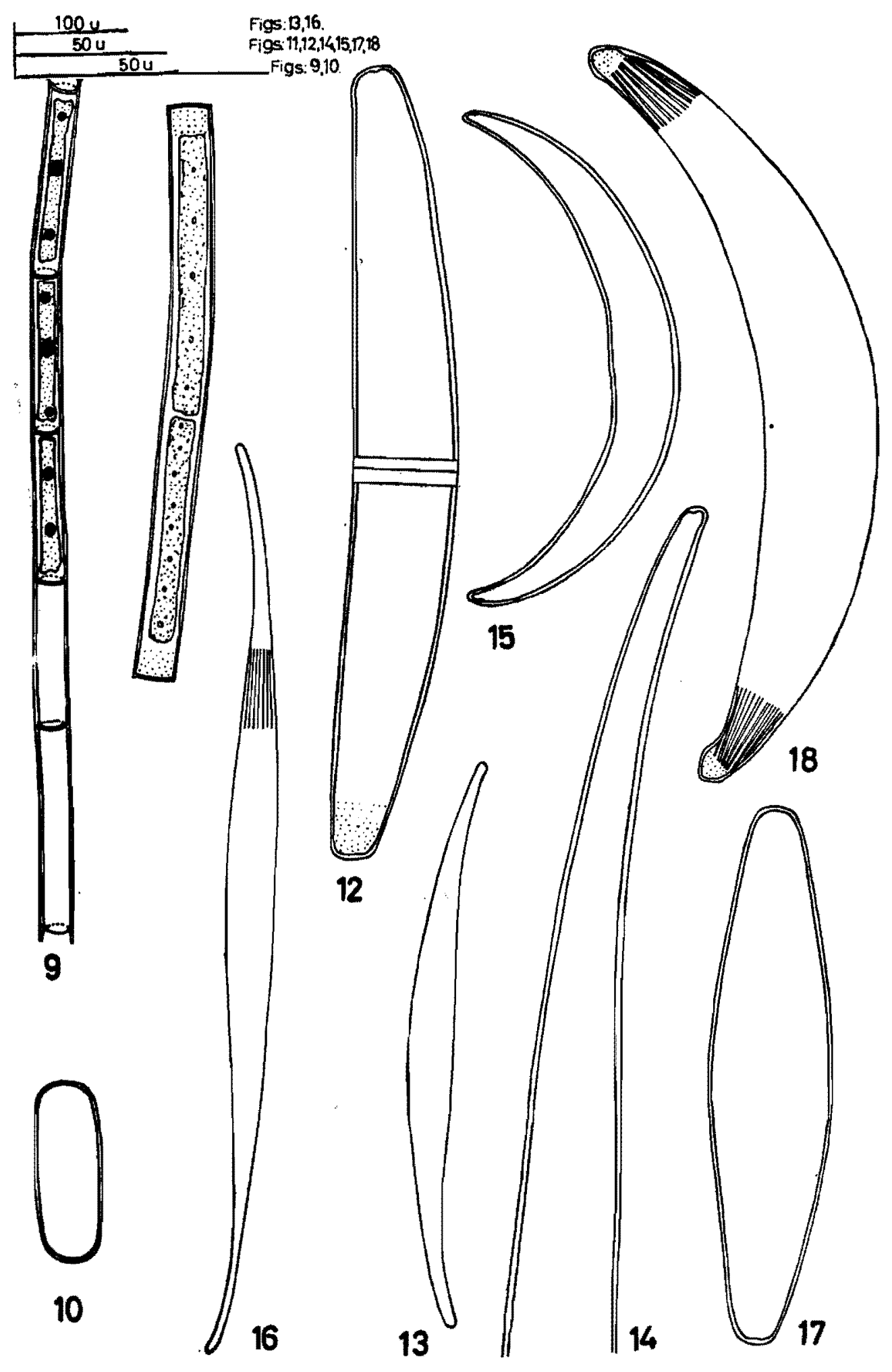




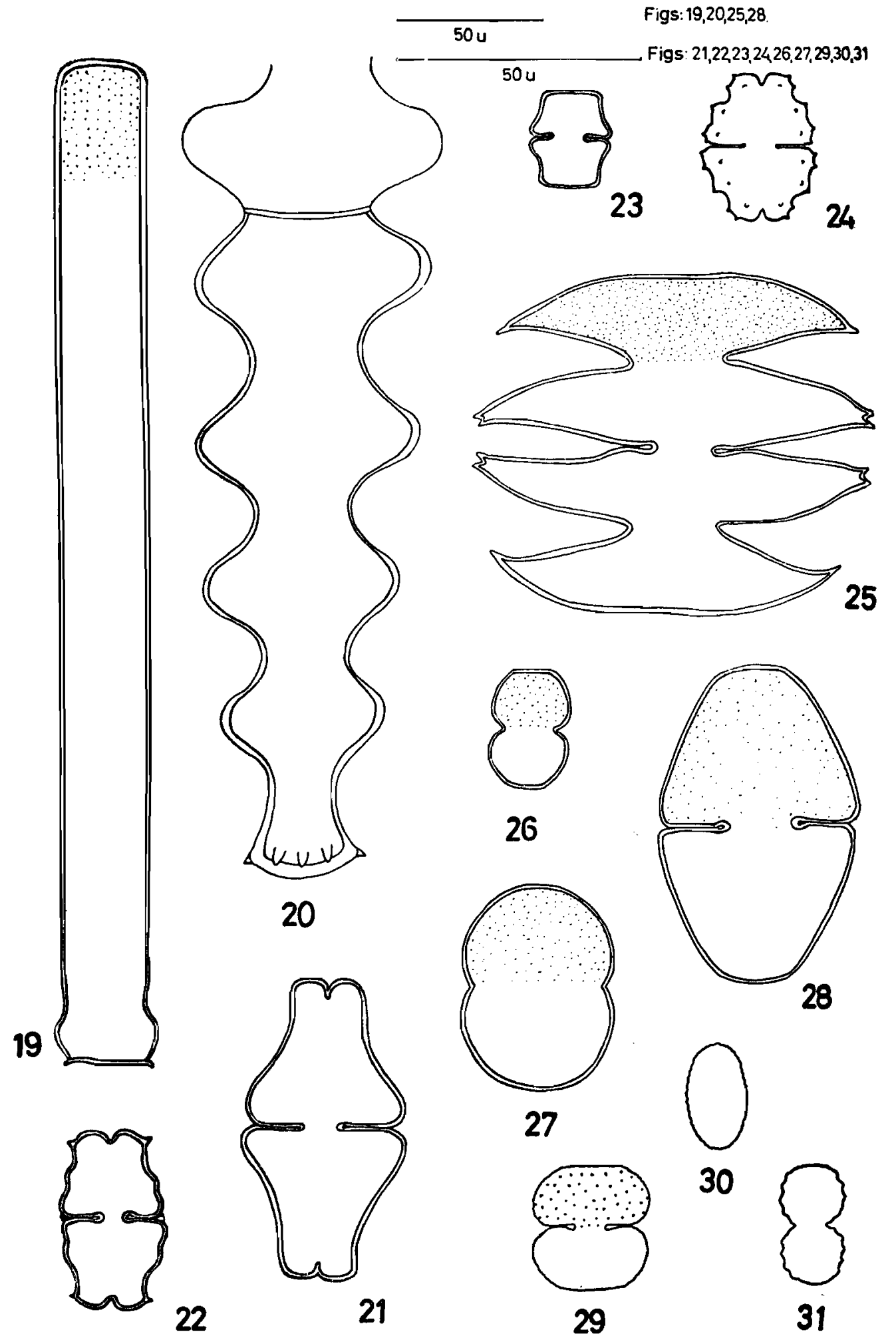




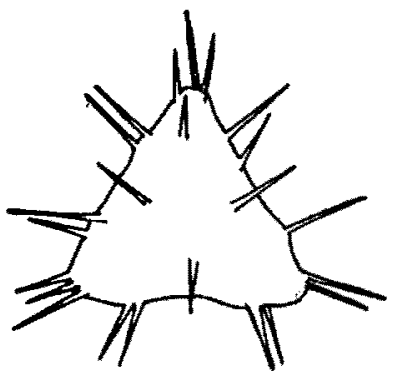

32
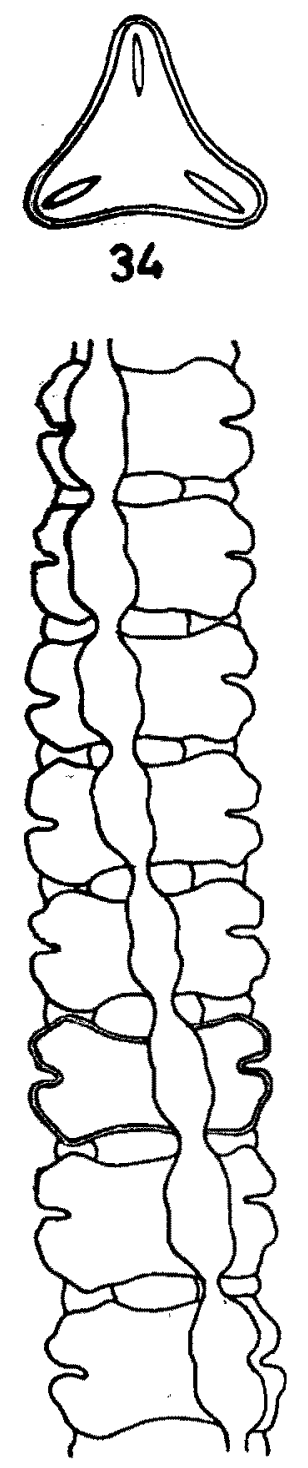

35

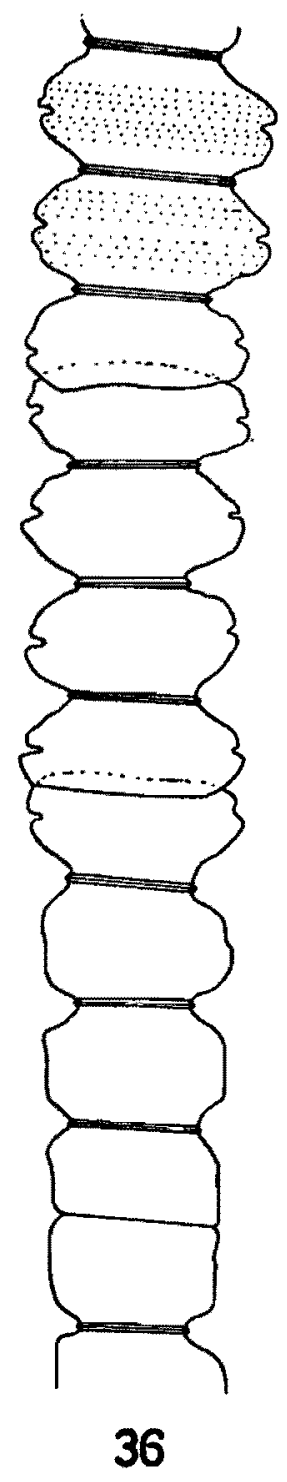

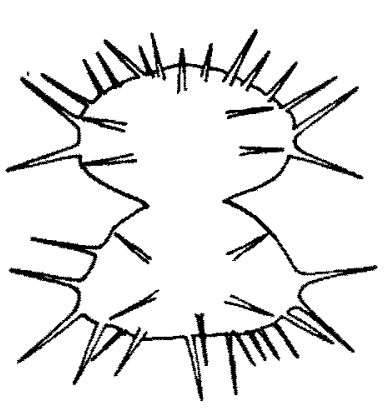

33

Figs. $32,33,34,35,36,37,38,39$
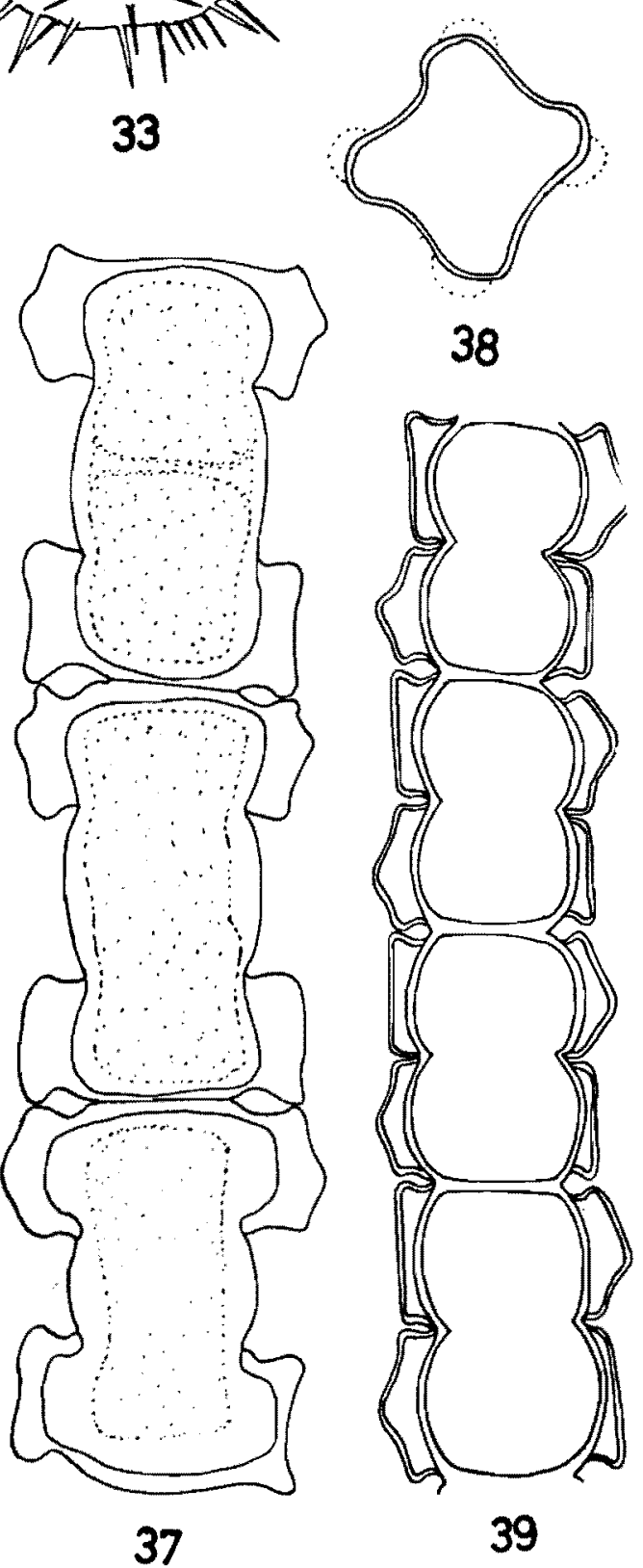


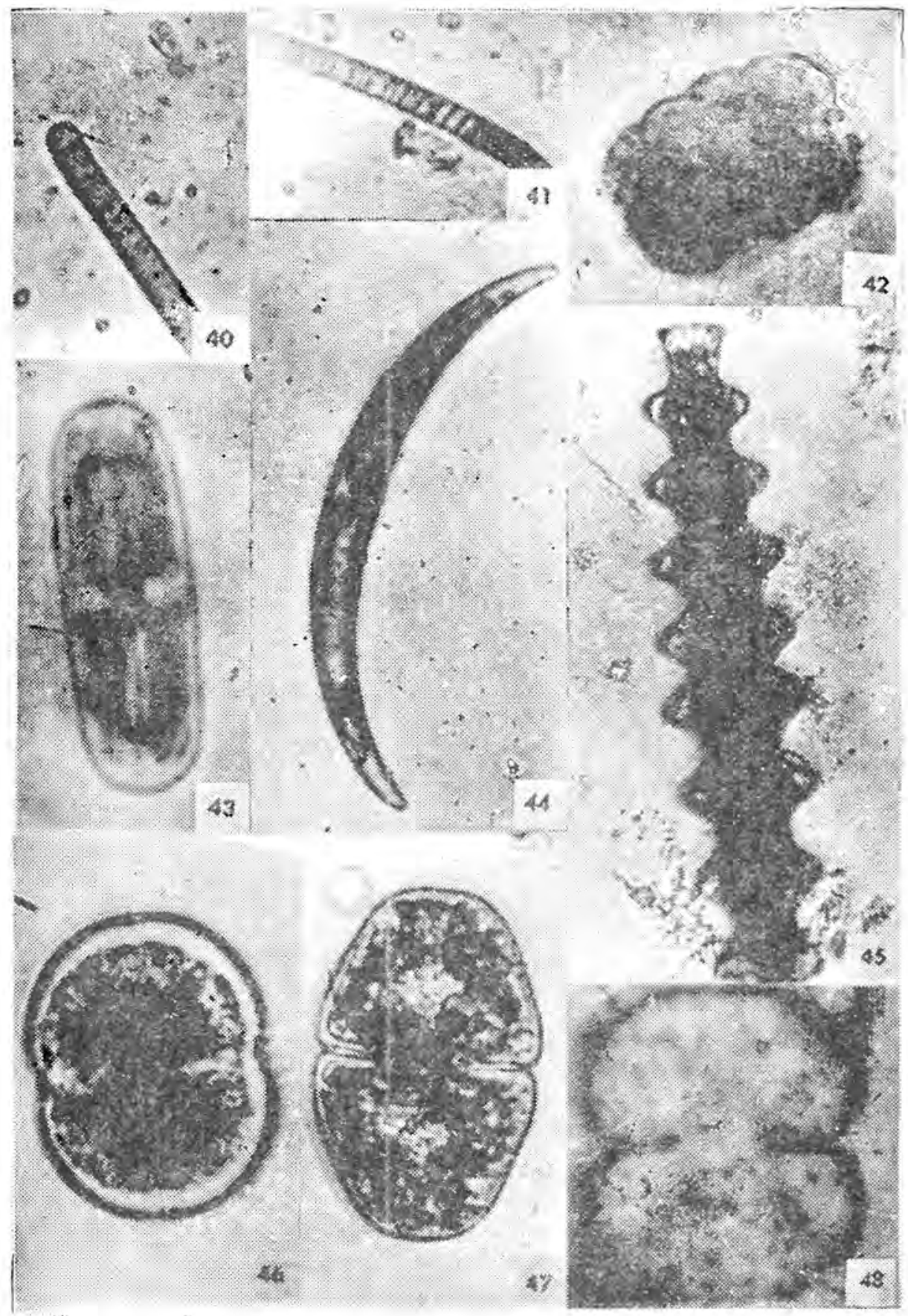




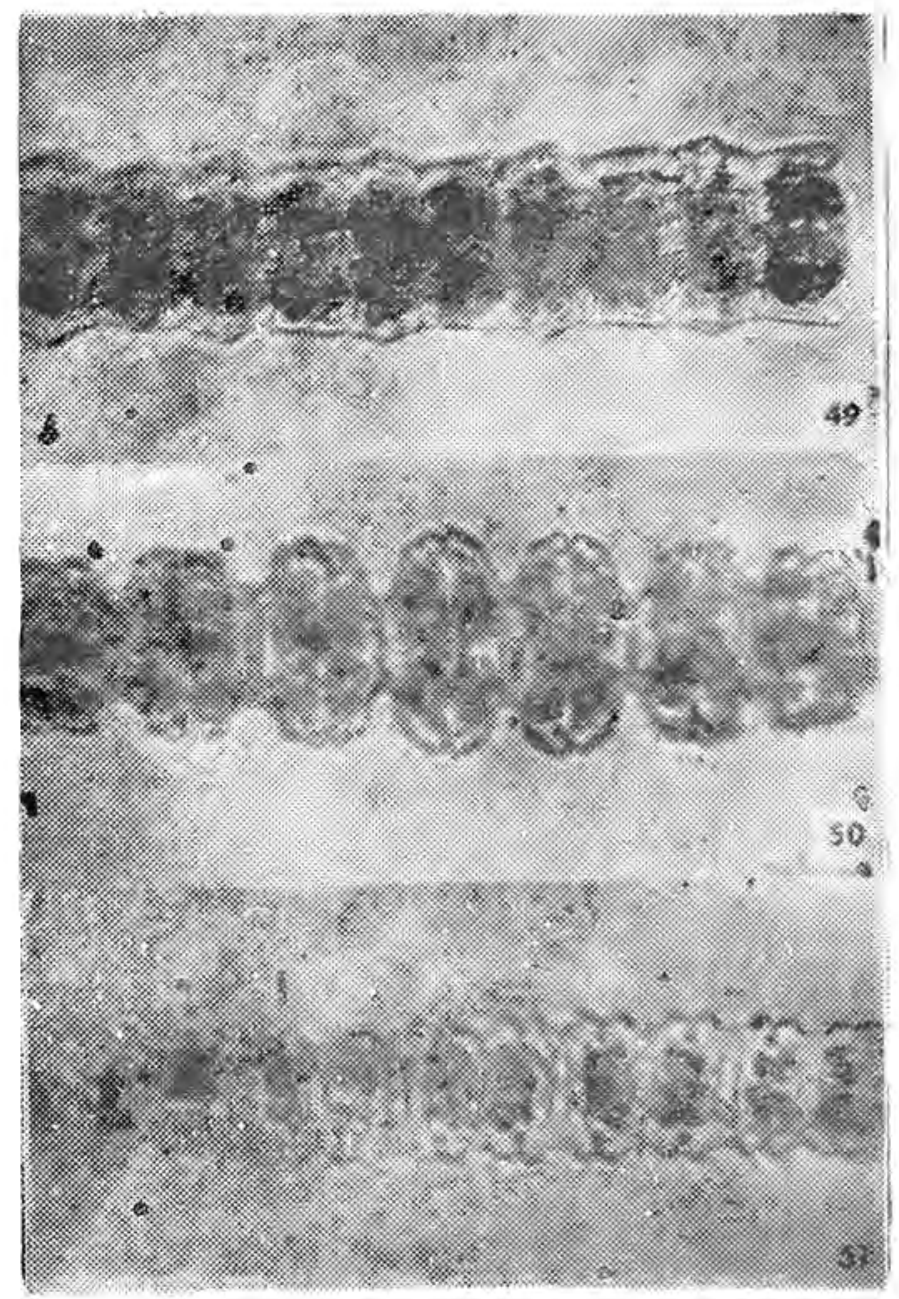

